\title{
Optimization of the Capsule Production Stochastic Cycle Time
}

\author{
Kamal Ghouchanian $^{1 *}$, Kiarash Ghassaban $^{2} \&$ Mojtaba Jokar ${ }^{3}$ \\ ${ }^{1}$ University of Sistan and Baluchestan, Iran \\ ${ }^{2}$ Department of Electrical Engineering, Amirkabir University of Technology, Tehran, Iran \\ ${ }^{3}$ Department of Chemical Engineering, Amirkabir University of Technology, Tehran, Iran \\ *Kamal Ghouchanian, E-mail: k.ghuchanian@yahoo.com
}

Received: June 16, 2017

Accepted: July 8, 2017

Online Published: July 13, 2017

doi:10.22158/rem.v2n4p21

URL: http://dx.doi.org/10.22158/rem.v2n4p21

\begin{abstract}
Manufacturing speed is one of the most important factors in pharmaceutical production, since the drug excipient is sensitive and its exposure to light and temperature should be controlled. Therefore, by minimizing the manufacturing cycle time, the quality of product can be improved. This process also results in minimizing the cost of manufacturing such as working hours, human resource, energy consumption and overhead cost while increasing the system productivity. In this study, using a stochastic dynamic programming method, the stochastic manufacturing cycle time of pharmaceutical product in a plant with process layout and concurrent machines is minimized. The result of this study has been compared to simulation modeling of the process.
\end{abstract}

\section{Keywords}

pharmaceutical product manufacturing, cycle time, product quality, stochastic dynamic optimization, process layout

\section{Introduction}

Over the past decade, there has been some progress in using systems engineering and optimization in pharmaceutical manufacturing industries. Affordable costs of healthcare demanded by governments as well as the competitive pharmaceutical industry has brought up the importance of manufacturing efficiency. Implementation of techniques such as work measurements and process engineering in manufacturing operations and product improvement have caught lots of attention which could potentially be even more beneficial with scheduling tools and process simulation (Papavasileiou, Koulouris, Siletti, \& Petrides, 2007). Pharmaceutical manufacturing processes can be enhanced using process modeling techniques both economically and reliably. Developing and improving manufacturing processes have recently raised significant complications in the pharmaceutical industry.

Nowadays, with the progress of new technologies, novel methods can potentially be used in the pharmaceutical industry in order to add more robustness and reliability to the outcomes and product 
quality. Well-designed manufacturing processes can lead to higher product quality and customer satisfaction. For example, contamination of manufacturing lines can be substantially reduced if semior fully-automated facilities were used in the processes, which in return leads to more robustness and reliability. On the other hand, by taking customer satisfaction into consideration using novel, robust and scientific techniques for measuring customer satisfaction see (Aguwa, Olya, \& Monplaisir, 2017), as well as process optimization and scheduling companies can provide agile manufacturing, an approach to customize the process without sacrificing the efficiency (O'Connor, Lawrence, \& Lee, 2016). Compared to other chemical technologies, manufacturing approaches used in pharmaceutical industries suffer from relatively low efficiency. Implementing such manufacturing technologies has been limiting the pharmaceutical manufacturing processes over the past few years (Myerson, Krumme, Nasr, Thomas, \& Braatz, 2015).

Primary and secondary manufacturing are key manufacturing phases in pharmaceutical industry. The primary phase involves active pharmaceutical ingredients production while secondary production concerns with inert materials and active pharmaceutical ingredients to make final products. Sarin, Sherali and Liao (2014) studied the secondary phase for optimizing the material flow which results in reduced direct labor hours, improved employee morale and reduced material handling costs as well as simplified flow of raw materials, reduced setup and make-span time and work-in-process inventories (Logendran, 1998). This method can assure a high operational flexibility, process train simplification through eliminating non-value-added and lengthy operations steps. With addressing this issue as the main purpose, the production environment addressed in this study is a manufacturing facility that produces capsules and tablets in small batches and employs semi-automatic material handling transporters to transfer items between different machine cells.

The remainder of this paper is structured as follows. In section 2, a literature review on pharmaceutical manufacturing process, cycle time and product make-span minimization and process scheduling as well as a review of using optimization methods in manufacturing industries have been discussed. Then in section 3, the implemented method has been explained and in section 4 , a case study of capsule production in a pharmaceutical manufacturing system has been discussed and the results are analyzed. The paper concludes with analyzing the results and future work directions.

\section{Literature Review}

As a result of the global competition, and due to a recent change in the general structure of the pharmaceutical industry, optimization of the pharmaceutical supply chains has caught considerable attention (Shah, 2004). Production planning taken place at the primary facility is of substantial value to the overall strength of this optimization process. Several studies have investigated different topics related to production planning and scheduling in process industries. For example, Kondili et al. (Kondili, Pantelides, \& Sargent, 1993) studied batch scheduling, Papageorgiou and Pantelides (1996) studied cyclic campaign planning and scheduling, integration of plant design and campaign scheduling 
(Fumero, Corsano, \& Montagna, 2011), in addition, Verderame and Floudas (2010), investigated process and demand uncertainty consideration into the model.

Furthermore, by applying time-based manufacturing techniques to two Dutch pharmaceutical plants, Brown and Vondrácek (Brown \& Vondráček, 2013) investigated the relationship between delivery dependability and throughput time. For multi-stage production processes, Kabra, Shaik and Rathore (2013) applied a State-Task Network-based mixed integer programming, by taking constraints, such as shelf-life limitations or late delivery penalties, into account. Using Radio Frequency Identification (RFID) technology, Pacciarelli and D'Ariano (2012) addressed data reliability issue in a case study performed on drug product manufacturing. Their conclusion states that the impact of increased data quality on the precision of production schedules is significant and can dramatically enhance productivity.

Recently using Automated Guided Vehicles (AGV) plays a significant role in material transferring in pharmaceutical manufacturing systems. But this model is barely studied when it comes to cycle time optimization in AGV-equipped manufacturing systems. One of the extensive research in this matter is done by Fazlollahtabar and Olya (2013). They proposed a novel stochastic optimization method for minimizing total cycle time in an AGV-equipped job shop manufacturing system. In addition to AGV's material handling time and machine's processing time, they considered AGV break-down. Also, they developed a cross-entropy approach when the process times follow different probability distributions. Their approach helps manufacturing systems to make processes more efficient through elimination of non-value-added material hold steps and streamlining the material transfer. So specifically, in pharmaceutical manufacturing process, this method can be beneficial to avoid drug contamination. Anwar and Nagi (1988) took the simultaneous scheduling of material handling transporters (e.g., automatic-guided vehicles or AGVs) and manufacturing equipment (e.g., machines and work-centers) into consideration in producing complex products. In their work, the cumulative lead time of the overall production schedule (i.e., total make-span) was minimized for on-time shipment as well as reduction in material-handling and inventory holding costs on the shop floor.

On the other hand, due to different reasons such as failures and costly maintenance, different types of uncertainties were encouraged to be taken into account in practice (Olya, Fazlollahtabar, \& Mahdavi, 2013). Since time and cost change as a random condition, and are uncertain, it is not practical to consider the arcs as deterministic values. In such cases, probability theory has been used to tackle the problem of randomness, and different groups have done lots of research on stochastic shortest path problem. When the arc lengths are random variables, the problem is subject to more complications (Olya, 2014b). Based on the study done by Frank (1969), this probability was the duration of the network shortest path is less than a specific value when link travel times are random and not time dependent variables. Mirchandani (1976) proposed another technique to obtain the shortest path distribution function in stochastic networks. It is not necessary to solve multiple integrals in this work. However, this approach can only be used for special cases where arc lengths are discrete random 
variables. Murthy and Sarkar (1966) showed that to identify the anticipated shortest path, the expected values of random link travel times can be used. This way, the problem becomes a deterministic shortest path problem thereby allowing the standard shortest path algorithms to be used to compute the anticipated shortest path in a static and stochastic network.

There are several studies in the literature discussing how to find a path with minimum expected value, variance and other criteria in stochastic networks. In these articles, the multi-criterion networks are evaluated. Fu and Rilett (1988) claimed that if the probability distribution for link delays was replaced by their expected values, it would lead to sub-optimal results. They proposed a dynamic programming strategy to solve this problem with conditional probability theory. But Olya (Olya, 2014a; Olya \& Fazlollahtabar, 2014) presented a stochastic dynamic method where the arc lengths are random variables with various probability density functions without replacing the random variables with their expected values. They solved the model using combinations of density functions while summing probability density functions in each step dynamically. For finding the shortest path in each step, they compared two probability density functions instead of two deterministic values in each step of dynamics programming. This method is more efficient than traditional methods such as maximum likelihood estimation and moment generating function in terms of computational effort. The proposed method generates an exact real-time solution with low computation cost. This method can be implemented in various real-life application ranging from healthcare systems optimization, operating room scheduling and robotics to air, train and ground routing and transportations as well as supply chain systems and can cater a solution to many healthcare and pharmaceutical-related issues in industry.

As it was discussed, the objective of this paper is to minimize pharmaceutical product make-span where the operation times are approximated by random variables with normal density function. We implemented the stochastic dynamic programming method proposed by Olya, Shirazi and Fazlollahtabar (2013) which is a more efficient approach to find the exact optimal solution under uncertainty. Using this method leads to improved product quality through dramatic decrease of product hold time by minimization of material handling and waiting times and elimination of multiple non-value-added transferring and hold steps.

\section{Methodology: Problem Definition and Modelling}

Generally, batch processing and semi-continuous production are utilized in drug manufacturing industry. In batch production system, each machine performs a particular task and there are different jobs. Considering the throughput of the manufacturing system and available resource and market-share, using identical parallel machines are allowed in job-shop manufacturing and process-flow systems where specific products are made and each job is different. This layout is used for manufacturing of small or medium size orders. Job shop layout consists of aggregating the machines based on their similarity in terms of nature of the task that they perform. Materials can be moved through the different 
department based on their production sequence. So, they may or may not go through certain operations and machines in the plant. The problem of job-shop scheduling is considered as a NP-Hard problem. Consider Figure 1 where the process flow and machine layout of tablet and capsules production system is depicted. It is clear that for each product, the material travels throughout the different areas according to its operation sequence. Operation sequence of two products (i.e., tablet and capsule) is shown in Figure 1. In this study, we consider tablet production as the main case. The objective is to minimize the make-span. There are different methods to minimize the product cycle time such as scheduling and optimization tools. In this study, a dynamic stochastic programming approach is used to minimize the make-span which results in increased productivity, reduced manufacturing cost and improved customer service. One important characteristic of any scheduling method is being dynamic due to uncertainty in the machine's processing time delay. In addition in many cases it is possible for the machine to break down. So, the method should be capable of minimizing the cycle time dynamically.

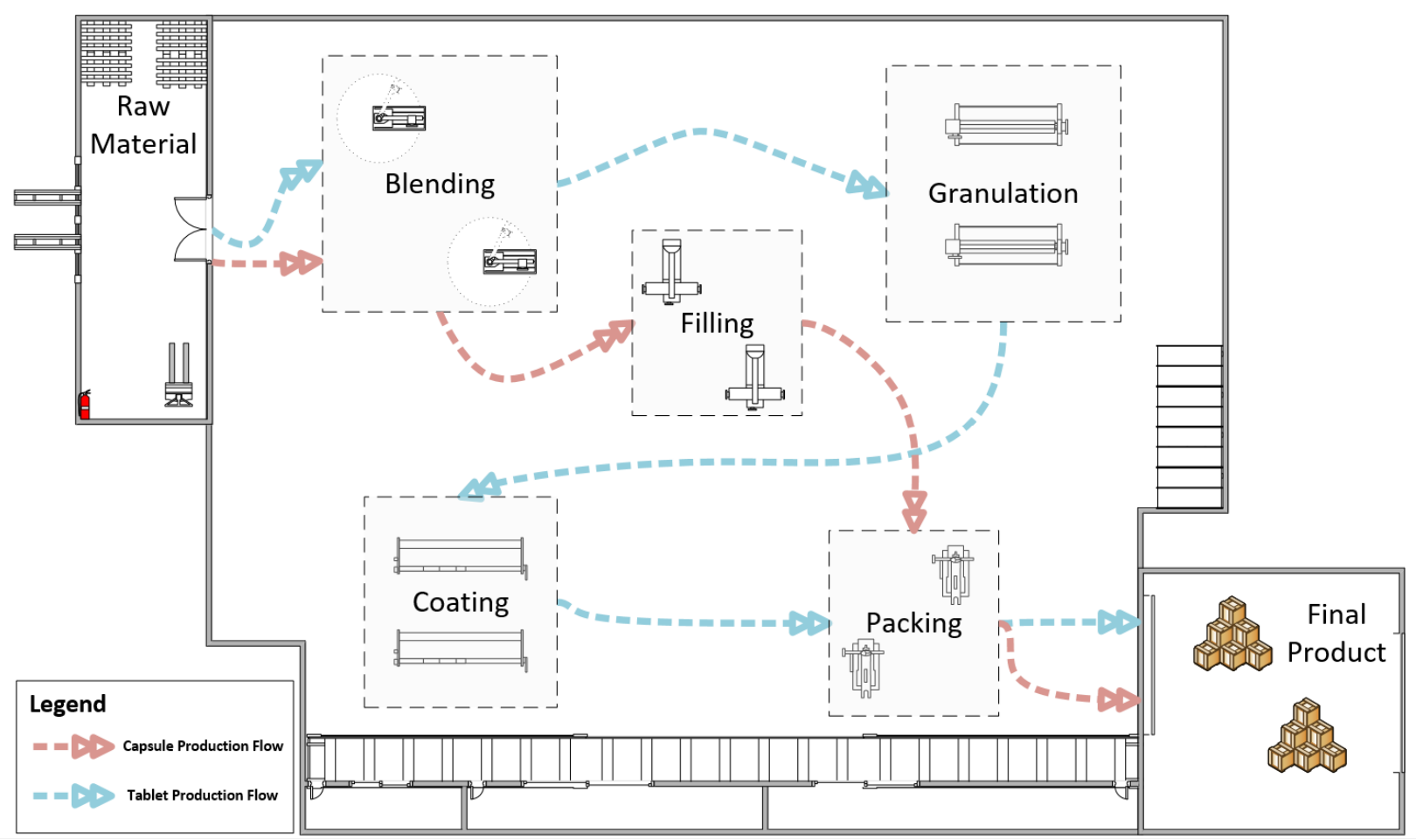

Figure 1. Process Flow and Machine Layout of Tablet and Capsules Production System

In this study operation time is considered as each individual task that consists of time of handling the material to the machine, delay time and machine processing time. So, aggregation of different operation times forms a make-span or product cycle time. Cycle time is the total time of the manufacturing process completion from the beginning to the end. In this research the time of operation is measured by normal distribution function instead of being considered as a deterministic value. That is because of the presence of uncertainty in the process and considering the correlation between processing time of all 
manufacturing jobs together.

Consider a plant floor as shown in Figure 1 which consists of a finite set of machines and products. The product flow forms a directed acyclic network which starts from raw material warehouse and finishes in final product warehouse. The operation time of each step is shown by an arrow and considered as a continuous normal random variable with parameters $\mu$ and $\sigma 2$. The objective is to minimize the total operation time (cycle time) of manufacturing each product.

\subsection{Operation Time Approximation}

Operation time is a stochastic variable which its precise prediction is of high importance in operation time optimization. Underestimating the operation time may result in long process delays, and overestimating the operation time yields low resource utilization rate. Therefore, we need a mechanism to predict the visit time. We may use a process flow data to predict the operation time. Here we use the historic data of the operation times for approximation.

Normal distribution is one of the most important distribution functions in statistics because it is simple to handle analytically, meaning that, it is possible to solve a large number of problems explicitly; the normal distribution is the result of the central limit theorem (Vazquez-Leal, Castaneda-Sheissa, Filobello-Nino, Sarmiento-Reyes, \& Sanchez Orea, 2012). The central limit theorem states that in a series of repeated observations, the precision of the approximation improves as the number of observations increases (Montgomery \& Runger, 2010). Besides, the bell shape of the normal distribution helps to model a variety of random variables in a practical way. The normal (or Gaussian) distribution integral has been widely used in manufacturing process modeling. The general formula for the probability density function of the normal distribution is

$$
f(\mathrm{x})=\frac{e^{-(x-u)^{2}} /\left(2 \sigma^{2}\right)}{\sigma \sqrt{2 \prod}}
$$

where $\mu$ is the location parameter and $\sigma$ is the scale parameter.

\subsection{Dynamic Programming Recurrence Function}

After approximating the operation times by different normal distributions, in order to find the minimum cycle time we used a backward dynamic programming approach proposed by Olya, Shirazi and Fazlollahtabar (2013) due to its high efficiency among the other methods. For modeling the cycle time minimization problem suppose that we have $\mathrm{M}$ machines in the manufacturing system and production flow forms an acyclic network. The optimal cycle time value function $T_{i}$ can be defined as the distribution of the shortest operation time from machine $i$ to machine M. The dynamic programming recurrence relation is defined in formula 1 (Olya et al., 2013).

$$
T_{i}=\min _{j>i}\left\lfloor d_{i j}+T_{j}\right\rfloor \quad \text { For } \quad i=\mathrm{M}-1
$$

To initiate the backward dynamic programming the boundary condition should be set as $T M=0$. For using the above recurrence function it is necessary to find the total distributions and compare them in 
each stage. The mechanism for calculating them is discussed in the next two sections.

\subsection{Calculating Sum of Two Density Functions}

Since the recurrence function includes adding up two random variables it necessitates the use of convolution to find the total operation time for each stage as it is described in theorem 1 (Olya et al., 2013).

Theorem 1: Let $X$ and $Y$ be two continuous random variables with density functions $f_{X}(x)$ and $f_{Y}(y)$, respectively. Assume that both $f_{X}(x)$ and $f_{Y}(y)$ are defined for all real numbers. Then the summation of two random variable $x$ and $y$ is a random variable with density function $f_{Z}(z)$, where $f_{Z}$ is the convolution of $f_{X}$ and $f_{Y}$.

$$
f_{\mathrm{z}}(\mathrm{z})=\int_{-\infty}^{+\infty} f_{x}(x) f_{y}(z-x) d x=\int_{-\infty}^{+\infty} f_{x}(z-y) f_{y}(y) d y
$$

For finding the summation of two normal random we know that if $X_{1}, X_{2}, \ldots, X_{n}$ are independent normal random variables with $\left(\mu_{1}, \sigma_{1}{ }^{2}\right),\left(\mu_{2}, \sigma_{2}^{2}\right), \ldots,\left(\mu_{n}, \sigma_{n}^{2}\right)$, then $Y=\sum_{i=1}^{n} X_{i}$ follows normal distribution with $\left(\sum_{i}^{n}=1 u_{i}, \sum_{i}^{n}=1 \ell_{i}^{2}\right)($ Olya et al., 2013).

\subsection{Comparing Two Density Functions}

Now we illustrate the method that we use to find the minimum between two normal random variables. In order to find the minimum random variable we calculate the probability that the first random variable $X_{1}$ with normal density function with $\left(\mu_{1}, \sigma_{1}^{2}\right)$ became smaller than the second random variable $X_{2}$ with normal density function with $\left(\mu_{2}, \sigma_{2}{ }^{2}\right)$ with considering above-mentioned result:

$$
P\left(\mathrm{X}_{1}<\mathrm{X}_{2}\right)=P\left(\mathrm{X}_{1}-\mathrm{X}_{2}\right)=P\left(\mathrm{Z}<\frac{0-\left(u_{1}-u_{2}\right)}{\sqrt{\sigma_{1}^{2}+\sigma_{2}^{2}}}\right)=\ell\left(\frac{u_{2}-u_{1}}{\sqrt{\sigma_{1}^{2}+\sigma_{2}^{2}}}\right)
$$

\section{Case Study and Results}

In pharmaceutical batch production, uncertainty can result from various sources that cause earliness and tardiness in the process. Consider the manufacturing network depicted in Figure 1. The process flow of two products capsules and tablets are illustrated in the Figure by red and blue arrows, respectively. We studied the capsule production in this work as it is illustrated in the Figure, raw materials are released by quality control and will be delivered to first stage of the process (i.e., blending) from the raw material warehouse. We consider raw material warehouse the origin of the network where the process begins. As it was indicated before, the operation time for each stage includes the material handling time delays and machine processing time. So, we consider each arrow as an indicator for operations. Following the definition of operation time, we defined 8 different operation times and considered the operation mapped network as an acyclic network. To form the network, we considered raw material warehouse as the origin, then moving toward blending stage. Since there are two 
machines, we will have two operation times. Then from each of which the material can choose one way among two available ways to move forward to the filling stage which also consists of two machines. There is a same situation for the product after being processed in filling stage. There are two different routes to go to the packing stage from each machine in filling stages. After being processed in packing stage the final product is ready to be delivered to customers. So, there is one raw material warehouse (RW), one final product warehouse (FW) and two machines in each department. The total number of the states is eight and the network has twelve arcs. This means that from each state except the destination, there are two possible ways to proceed to the next state. As explained before, this procedure can be modeled as an acyclic network in which arc lengths are normal random variables. The operation times for each stage are measured in minutes and since the operation times are not deterministic, using the Arena software we approximate the density functions of each operation time as represented in Table 1.

Table 1. Normal Density Function Approximation of Operation Time at each Stage

\begin{tabular}{lllll}
\hline No. & State No. & Stage & Machine sequence & Approximation \\
\hline 1 & 1 & Blending & RW-B1 & $\mathrm{N}(104,2)$ \\
2 & 1 & Blending & RW-B2 & $\mathrm{N}(129,12)$ \\
3 & 2 & Filling & B1-F1 & $\mathrm{N}(165,9)$ \\
4 & 2 & Filling & B1-F2 & $\mathrm{N}(170,4)$ \\
5 & 3 & Filling & B2-F1 & $\mathrm{N}(145,3)$ \\
6 & 3 & Filling & B2-F2 & $\mathrm{N}(140,3)$ \\
7 & 4 & Packing & F1-P1 & $\mathrm{N}(155,3)$ \\
8 & 4 & Packing & F1-P2 & $\mathrm{N}(140,2)$ \\
9 & 5 & Packing & F2-P1 & $\mathrm{N}(120,5)$ \\
10 & 5 & Packing & F2-P2 & $\mathrm{N}(128,3)$ \\
11 & 6 & Final transfer & P1-FW & $\mathrm{N}(20,2)$ \\
12 & 7 & Final transfer & P2-FW & $\mathrm{N}(25,3)$ \\
\hline
\end{tabular}

After finding the density function of each operation time, the manufacturing sequence that has the lowest total duration has been found by using the stochastic dynamic method where the network origin is raw material warehouse and the network destination is the final product warehouse. In order to find the shortest cycle time, we need to find the total operation time for each stage by using formula 1 as well as the probability that a certain sequence of operations has the shortest total operation time. In order to begin the backward dynamic programming, the boundary condition is set to zero $\left(T_{8}=0\right)$. The result of applying the method is represented in Table 2. 
Table 2. Stepwise Result of Dynamic Programming

\begin{tabular}{llll}
\hline State No. & Selected operation sequence & Operation time & Probability \\
\hline 8 & FW & - & - \\
7 & P2-FW & $\mathrm{N}(25,3)$ & - \\
6 & P1-FW & $\mathrm{N}(20,2)$ & - \\
5 & F2-P1-FW & $\mathrm{N}(140,7)$ & 0.99 \\
4 & F1-P2-FW & $\mathrm{N}(165,5)$ & 0.99 \\
3 & B2-F2-P1-FW & $\mathrm{N}(280,10)$ & 0.99 \\
2 & B1-F2-P1-FW & $\mathrm{N}(310,11)$ & 0.99 \\
1 & RW-B2-F2-P1-FW & $\mathrm{N}(409,22)$ & 0.80 \\
\hline
\end{tabular}

As it is indicted in Table 2 the manufacturing route with the shortest cycle time of the capsule manufacturing is the sequence that starts from the raw material warehouse, goes to machine number 2 in the blending section, then it goes to machine number 2 in filling, then goes to machine number 1 in the packing section and eventually goes to final product warehouse. The probability that this sequence has the lowest cycle time is 0.8 . So, it can be stated that this sequence has the minimum cycle time with a probability of 80 percent.

For validating the results, we have used the process simulation in Arena software. The results of the process simulation are represented in Table 3.

Table 3. Operation Time Simulation Result

\begin{tabular}{llll}
\hline Sequence & Average & Minimum & Maximum \\
\hline RW-B1-F1-P1-FW & 444.2216 & 432.7189 & 457.0553 \\
RW-B1-F1-P2-FW & 449.3251 & 438.1263 & 461.3951 \\
RW-B1-F2-P1-FW & 413.8709 & 401.4177 & 426.1416 \\
RW-B1-F2-P2-FW & 426.6687 & 416.8918 & 435.9161 \\
RW-B2-F1-P1-FW & 449.0533 & 437.2045 & 461.1022 \\
RW-B2-F1-P2-FW & 439.7201 & 426.4335 & 449.4967 \\
RW-B2-F2-P1-FW & 409.4163 & 394.547 & 425.6678 \\
RW-B2-F2-P2-FW & 421.8703 & 410.4095 & 434.8484 \\
\hline
\end{tabular}

Considering the results of simulation in Table 3, it can be concluded that the results of simulation support the result of the implemented method in this research. 


\section{Conclusions}

Although pharmaceutical manufacturing optimization is very important in this sensitive industry, it has not been studied very well using progressed techniques. Accelerating the pharmaceutical manufacturing process is essential for manufacturers to remain as a strong competitor in this emerging industry. Cycle time optimization is considered as an important issue in manufacturing processes, especially in the pharmaceutical industry where the raw material is sensitive and needs to be proceeded rapidly. The problem becomes more complicated due to uncertainty of the operation times. To tackle this issue, a stochastic dynamic programming method has been used where the operation times are approximated by normal density functions. The results of implementing the mentioned method are compared with the process simulation results. Using this method is beneficial for pharmaceutical and healthcare processes since it is more realistic because of considering the probability density function of each operation time instead of their expected values. Using the expected values decreases the level of uncertainty of the model. Using new technologies such as continuous production can improve the process speed; however, using the new technology needs the cost benefit analysis and high initial investment for implementing the technology in the plant.

\section{References}

Aguwa, C., Olya, M. H., \& Monplaisir, L. (2017). Modeling of fuzzy-based voice of customer for business decision analytics. Knowledge-Based Systems, 125, 136-145. https://doi.org/10.1016/j.knosys.2017.03.019

Anwar, M. F., \& Nagi, R. (1998). Integrated scheduling of material handling and manufacturing activities for just-in-time production of complex assemblies. International Journal of Production Research, 36(3), 653-681. https://doi.org/10.1080/002075498193624

Brown, S., \& Vondráček, P. (2013). Implementing time-based manufacturing practices in pharmaceutical preparation manufacturers. Production Planning \& Control, 24(1), 28-46. https://doi.org/10.1080/09537287.2011.598267

Fazlollahtabar, H., \& Olya, M. H. (2013). A cross-entropy heuristic statistical modeling for determining total stochastic material handling time. The International Journal of Advanced Manufacturing Technology, 67(5-8), 1631-1641. https://doi.org/10.1007/s00170-012-4596-z

Frank, H. (1969). Shortest paths in probabilistic graphs. Operations Research, 17(4), 583-599. https://doi.org/10.1287/opre.17.4.583

Fu, L., \& Rilett, L. R. (1998). Expected shortest paths in dynamic and stochastic traffic networks. Transportation Research Part B: Methodological, 32(7), 499-516. https://doi.org/10.1016/S0191-2615(98)00016-2

Fumero, Y., Corsano, G., \& Montagna, J. M. (2011). Detailed design of multiproduct batch plants considering production scheduling. Industrial \& Engineering Chemistry Research, 50(10), 
6146-6160. https://doi.org/10.1021/ie1008376

Kabra, S., Shaik, M. A., \& Rathore, A. S. (2013). Multi-period scheduling of a multi-stage multi-product bio-pharmaceutical process. Computers \& Chemical Engineering, 57, 95-103. https://doi.org/10.1016/j.compchemeng.2013.03.009

Kondili, E., Pantelides, C. C., \& Sargent, R. W. H. (1993). A general algorithm for short-term scheduling of batch operations-I. MILP formulation. Computers \& Chemical Engineering, 17(2), 211-227. https://doi.org/10.1016/0098-1354(93)80015-F

Logendran, R. (1998). Group technology and cellular manufacturing: Methodologies and applications (Vol. 1). Taylor \& Francis.

Mirchandani, P. B. (1976). Shortest distance and reliability of probabilistic networks. Computers \& Operations Research, 3(4), 347-355. https://doi.org/10.1016/0305-0548(76)90017-4

Montgomery, D. C., \& Runger, G. C. (2010). Applied statistics and probability for engineers. John Wiley \& Sons.

Murthy, I., \& Sarkar, S. (1996). A relaxation-based pruning technique for a class of stochastic shortest path problems. Transportation Science, 30(3), 220-236. https://doi.org/10.1287/trsc.30.3.220

Myerson, A. S., Krumme, M., Nasr, M., Thomas, H., \& Braatz, R. D. (2015). Control systems engineering in continuous pharmaceutical manufacturing. May 20-21, 2014 Continuous Manufacturing Symposium. Journal of Pharmaceutical Sciences, 104(3), 832-839. https://doi.org/10.1002/jps.24311

O’Connor, T. F., Lawrence, X. Y., \& Lee, S. L. (2016). Emerging technology: A key enabler for modernizing pharmaceutical manufacturing and advancing product quality. International Journal of Pharmaceutics, 509(1), 492-498. https://doi.org/10.1016/j.ijpharm.2016.05.058

Olya, M. H. (2014a). Applying Dijkstra's algorithm for general shortest path problem with normal probability distribution arc length. International Journal of Operational Research, 21(2), 143. https://doi.org/10.1504/IJOR.2014.064541

Olya, M. H. (2014b). Finding shortest path in a combined exponential-gamma probability distribution arc length. International Journal of Operational Research, 21(1), 25. https://doi.org/10.1504/IJOR.2014.064020

Olya, M. H., \& Fazlollahtabar, H. (2014). Finding Shortest Path in a Combined Exponential-Gamma-Normal Probability Distribution Arc Length. Advances in Industrial Engineering and Management, 3(4), 3544.

Olya, M. H., Fazlollahtabar, H., \& Mahdavi, I. (2013). Shortest Path Problem with Gamma Probability Distribution Arc Length. International Journal of Applied Operational Research, 2(4), 55-66. Retrieved from http://www.ijorlu.liau.ac.ir/article-1-208-en.html

Olya, M. H., Shirazi, B., \& Fazlollahtabar, H. (2013). Adapted Dynamic Program to Find Shortest Path in a Network having Normal Probability Distribution Arc Length. Advances in Industrial Engineering and Management, 2(1), 5-10. 
Pacciarelli, D., \& D'Ariano, A. (2012). Increasing the reliability of production schedules in a pharmaceutical packaging department. Journal of Medical Systems, 36(6), 3407-3421. https://doi.org/10.1007/s10916-011-9791-1

Papageorgiou, L. G., \& Pantelides, C. C. (1996). Optimal campaign planning/scheduling of multipurpose batch/semicontinuous plants. 1. Mathematical formulation. Industrial \& Engineering Chemistry Research, 35(2), 488-509. https://doi.org/10.1021/ie9500811

Papavasileiou, V., Koulouris, A., Siletti, C., \& Petrides, D. (2007). Optimize manufacturing of pharmaceutical products with process simulation and production scheduling tools. Chemical Engineering Research and Design, 85(7), 1086-1097. https://doi.org/10.1205/cherd06240

Sarin, S. C., Sherali, H. D., \& Liao, L. (2014). Primary pharmaceutical manufacturing scheduling problem. IIE Transactions, 46(12), 1298-1314. https://doi.org/10.1080/0740817X.2014.882529

Shah, N. (2004). Pharmaceutical supply chains: Key issues and strategies for optimisation. Computers \& Chemical Engineering, 28(6), 929-941. https://doi.org/10.1016/j.compchemeng.2003.09.022

Vazquez-Leal, H., Castaneda-Sheissa, R., Filobello-Nino, U., Sarmiento-Reyes, A., \& Sanchez Orea, J. (2012). High accurate simple approximation of normal distribution integral. Mathematical Problems in Engineering, 2012. https://doi.org/10.1155/2012/124029

Verderame, P. M., \& Floudas, C. A. (2010). Integration of operational planning and medium-term scheduling for large-scale industrial batch plants under demand and processing time uncertainty. Industrial \& Engineering Chemistry Research, 49(10), 4948-4965. https://doi.org/10.1021/ie901973e 